\title{
Desempenho balístico de estruturas multicamadas à base de alumina/epóxi
}

\section{(Ballistic performance of multilayer structures based on alumina/epoxy)}

\author{
M. B. Polla ${ }^{1 *}$, D. C. N. Fabris ${ }^{1}$, A. De Noni Jr. ${ }^{2}$, O.R. K. Montedo ${ }^{1}$ \\ ${ }^{1}$ Universidade do Extremo Sul Catarinense, Programa de Pós-Graduação em Ciência e Engenharia de \\ Materiais, Av. Universitário 1105, 88806-000, Criciúma, SC, Brasil \\ ${ }^{2}$ Universidade Federal de Santa Catarina, Departamento de Engenharia Química e Engenharia de Alimentos, \\ Florianópolis, SC, Brasil
}

\begin{abstract}
Resumo
Alumina é comumente utilizada em blindagens compostas. Apresenta baixa tenacidade à fratura e esta propriedade está relacionada à resistência a múltiplos disparos. Com o intuito de melhorar a durabilidade em campo uma alternativa seria utilizar cerâmicas multicamadas. Neste estudo foi avaliado o desempenho balístico de estruturas multicamadas à base de alumina/epóxi e o efeito da adição de fibra de vidro como material de reforço. Os resultados foram comparados com placas de alumina individual de mesma espessura total que os sistemas multicamadas. $\mathrm{O}$ desempenho balístico dos painéis em mosaico de $\mathrm{Al}_{2} \mathrm{O}_{3} / \mathrm{Al}$ foi avaliado por meio de ensaio balístico DOP (depth of penetration) com projétil 7,62 mm perfurante. Os resultados indicaram que as placas individuais apresentaram melhor desempenho. No caso das estruturas $\mathrm{Al}_{2} \mathrm{O}_{3}$ /epóxi, a resistência balística diminuiu com a redução da espessura das lâminas de alumina e com o aumento da camada de epóxi. A fibra de vidro não apresentou efeito significativo no desempenho. Palavras-chave: blindagem cerâmica, alumina, estrutura multicamadas, desempenho balístico.
\end{abstract}

Abstract

Alumina is commonly used in composite shields. It presents low fracture toughness and this property is related to resistance to multiple shots. In order to improve the lifetime in applications, an alternative would be the use of multilayer ceramics. This study evaluated the ballistic performance of multilayer aluminalepoxy structures and the effect of glass fiber as a reinforcing material. The results were compared to individual alumina plates of the same total thickness as multilayer systems. The ballistic performance of the $\mathrm{Al}_{2} \mathrm{O}_{3} / \mathrm{Al}$ mosaic panels was evaluated using a DOP (depth of penetration) ballistic test with the $7.62 \mathrm{~mm}$ armor-piercing projectile. The results indicated that the individual plate showed better performance. In the case of $\mathrm{Al}_{2} \mathrm{O}_{3}$ /epoxy structures, the ballistic resistance decreased with the reduction of the thickness of the alumina layer and with the increase of the epoxy layer. The glass fiber showed no improvement in ballistic performance.

Keywords: ceramic shielding, alumina, multilayer structure, ballistic performance.

\section{INTRODUÇÃO}

Materiais cerâmicos apresentam elevada dureza, resistência à compressão e baixa densidade em relação aos materiais metálicos. Tais propriedades qualificam as cerâmicas para aplicações em blindagem balística. O desenvolvimento de blindagens cerâmicas tornou os sistemas de proteção mais leves e eficientes em comparação com as blindagens metálicas convencionais [1-3]. As blindagens cerâmicas geralmente são compostas por duas partes: uma camada de cerâmica apoiada e uma placa de apoio metálica (liga de alumínio ou aço) ou um polímero reforçado com fibra [4]. A cerâmica constitui a primeira camada da armadura, sua função é fragmentar o projétil e absorver a energia inicial do impacto. A segunda camada é

*marianaborgespolla@hotmail.com

(D) https://orcid.org/0000-0003-2344-1987 formada pelo material dúctil e tem a função de absorver a energia cinética residual do projétil, conter os fragmentos de projétil e cerâmica, evitando deformações excessivas a fim de garantir a integridade do sistema protegido [5]. Atualmente os materiais cerâmicos mais utilizados em blindagens são o carbeto de boro $\left(\mathrm{B}_{4} \mathrm{C}\right)$ e o carbeto de silício $(\mathrm{SiC})$ para calibres pesados, e a alumina $\left(\mathrm{Al}_{2} \mathrm{O}_{3}\right)$ para calibres leves [5]. Dentre as cerâmicas técnicas, a alumina é comumente utilizada devido à sua maior disponibilidade e melhor relação custo/benefício. No entanto, a alumina apresenta baixa tenacidade à fratura, absorvendo pequena quantidade de energia antes de sua fratura $[2,5,6]$.

Em blindagens mistas cerâmica/metal, os danos causados pelo primeiro projétil reduzem significativamente o desempenho do sistema de proteção contra demais disparos [7]. Com o intuito de melhorar o desempenho balístico da blindagem, e consequentemente melhorar a durabilidade em campo, uma alternativa poderia ser a utilização de cerâmicas 
multicamadas [8-11]. Em cerâmicas multicamadas ocorre o desvio da trajetória de trincas camada após camada, devido à presença de interfaces, o que resulta no aumento da absorção de energia [12]. As cerâmicas em camadas têm maior resistência a múltiplos impactos comparadas com cerâmicas monolíticas e a área danificada é reduzida consideravelmente com o aumento do número de camadas, pois a absorção de energia é melhorada [9]. A propagação do dano ao longo da blindagem também pode ser reduzida utilizando-se mosaicos ao invés de placas cerâmicas monolíticas. Dessa forma, quando o painel é atingindo o dano fica concentrado apenas na peça atingida e nas adjacentes [13-15]. De acordo com a literatura [16], o confinamento da cerâmica melhora o desempenho balístico do sistema de proteção. Observase que durante o processo de penetração da cerâmica não confinada o projétil permanece com energia suficiente para perfurar a placa de apoio. Com o confinamento a perfuração do suporte é reduzida ou eliminada. Durante o processo de penetração os fragmentos são ejetados na direção oposta do impacto. O confinamento lateral limita a deformação da cerâmica no sentido radial, o que proporciona a desaceleração do projétil de modo mais eficaz. Jiusti et al. [17] avaliaram a profundidade de penetração em aluminas em painéis de mosaico com diferentes tipos de materiais de rejunte. Painéis em mosaico preenchidos com epóxi apresentaram os melhores resultados e os danos permaneceram limitados à placa atingida. Quanto à geometria, placas hexagonais são mais eficientes na atenuação dos efeitos de borda em comparação com placas quadradas. Estas placas apresentam maior vulnerabilidade criada pelos pontos de contato que ficam localizados entre quatro peças [6]. Por outro lado, são as mais utilizadas por apresentar maior facilidade de produção. Com o intuito de reduzir a maior vulnerabilidade das placas quadradas, uma alternativa é fixa-las ao suporte com defasagem de meia peça. Assim, o ponto de junção fica entre três placas ao invés de quatro [18].

Neste estudo o desempenho balístico foi avaliado em função do número de camadas de alumina ( 2 camadas de $3 \mathrm{~mm}$ de espessura e 3 camadas de $2 \mathrm{~mm}$ de espessura) e da espessura da camada intermediaria ( 0,5 e $1 \mathrm{~mm}$ de epóxi). Os resultados foram comparados com placas de alumina monolíticas $(6 \mathrm{~mm}$ de espessura). Foi também avaliado o efeito da adição de fibra de vidro como material de reforço da camada de epóxi. Foram confeccionados sistemas em mosaico com placas quadradas $\left(\mathrm{Al}_{2} \mathrm{O}_{3}\right)$. O desempenho balístico dos painéis $\mathrm{Al}_{2} \mathrm{O}_{3} / \mathrm{Al}$ foi avaliado por meio de ensaio balístico DOP (depth of penetration) com projétil $7,62 \mathrm{~mm}$ perfurante, conforme norma NIJ 0108.01 (nível IV).

\section{PROCEDIMENTO EXPERIMENTAL}

Produção das placas alvo cerâmicas: na confecção das placas cerâmicas foi utilizado pó de alumina com 99,8\% de pureza, área de superfície BET de $4,9 \mathrm{~m}^{2} \cdot \mathrm{g}^{-1}$ e tamanho médio de partícula de 1,7 $\mu \mathrm{m}$ (Almatis). A preparação do pó foi feita com adição de $10 \%$ de água deionizada, $0,6 \%$ de ligante (PVA) e $0,4 \%$ de plastificante (DEG - dietilenoglicol) com auxílio de um pulverizador. Em seguida o pó foi passado em peneira com abertura de $710 \mu \mathrm{m}$, para homogeneizar a umidade e auxiliar na granulação. Com o intuito de melhorar a compactação e esfericidade dos grânulos, a massa foi colocada em recipiente polimérico e mantida em rotação durante $1 \mathrm{~h}$, utilizando um moinho girajarros laboratorial. Em seguida os grânulos foram passados em peneira de $300 \mu \mathrm{m}$, para limitar o tamanho [19]. O pó foi seco em estufa por $2 \mathrm{~h}$ a $110{ }^{\circ} \mathrm{C}$. Após o resfriamento adicionaram-se $2 \%$ de umidade por pulverização e o pó foi mantido em repouso por $8 \mathrm{~h}$. As placas de alumina foram conformadas pelo processo de prensagem uniaxial, utilizando uma prensa hidráulica laboratorial (Gabbrielli, GT 0785), estampo de $60 \times 120 \mathrm{~mm}^{2}$ e pressão total de $140 \mathrm{MPa}$. Confeccionaram-se peças com 2,26, 3,39 e $6,78 \mathrm{~mm}$ de espessura. Após a prensagem e secagem as peças foram cortadas em dimensões de $56,5 \times 56,5 \mathrm{~mm}^{2}$. A sinterização ocorreu em forno laboratorial (Fortelab, FE 1700 V 200) a uma temperatura máxima de $1670{ }^{\circ} \mathrm{C}$, taxa de aquecimento de $10{ }^{\circ} \mathrm{C} / \mathrm{min}$ e tempo de permanência na máxima temperatura de $3 \mathrm{~h}$. Após a sinterização as peças ficaram com $50 \mathrm{~mm}$ de lado e 2,0,3,0 e 6,0 $\mathrm{mm}$ de espessura.

Produção das placas de confinamento: as placas de confinamento foram produzidas utilizando pó de alumina com 92,2\% de pureza (linha Granalox, Nabaltec). A etapa de preparação do pó não foi realizada, pois a alumina já estava granulada de fábrica e pronta para prensagem. A etapa de conformação das placas de confinamento foi igual à descrita anteriormente; as espessuras das placas foram as mesmas da placa monolítica e das espessuras totais das estruturas multicamadas (Tabela I). Foram produzidas peças com 7,02, 7,60, 7,78, 8,19, 8,54 e 9,36 mm de espessura. Após a prensagem e secagem as peças foram cortadas em dimensões de $58,5 \times 58,5 \mathrm{~mm}^{2}$. Na sinterização foi utilizada temperatura máxima de $1580{ }^{\circ} \mathrm{C}$ por $2 \mathrm{~h}$ e taxa de aquecimento de $10{ }^{\circ} \mathrm{C} / \mathrm{min}$. Após a sinterização as peças ficaram com $50 \mathrm{~mm}$ de lado e $6,0,6,5,6,65,7,0$, 7,3 e $8,0 \mathrm{~mm}$ de espessura.

Preparação dos suportes de alumínio: as placas de suporte foram confeccionadas a partir de tarugos cilíndricos de alumínio liga 6063, com diâmetro de $208 \mathrm{~mm}$ e espessura de $50 \mathrm{~mm}$ (Alumasa). O alumínio é comumente utilizado em blindagens por apresentar menor densidade em relação ao aço e ser facilmente obtido. A capacidade de penetração do projétil na placa de alumínio diminui com o aumento da dureza. Com o intuito de aumentar a dureza das placas de apoio, os tarugos foram submetidos a tratamento térmico [20]. Este tratamento constituiu em uma primeira etapa de solubilização seguida por uma etapa de envelhecimento visando obter dureza de aproximadamente $73 \mathrm{HB}$ [21]. Na etapa de solubilização a temperatura utilizada foi de $520{ }^{\circ} \mathrm{C}$ com um tempo de permanência de $2 \mathrm{~h}$. O resfriamento ocorreu em um tanque de água à temperatura ambiente. $\mathrm{O}$ envelhecimento foi realizado em estufa a $175^{\circ} \mathrm{C}$ durante $7 \mathrm{~h}$. Após o tratamento a dureza Brinell foi medida de acordo com a norma ASTM E10 em durômetro Brinell (Panantec). Foram realizadas três impressões em cada tarugo. 
Preparação dos painéis balísticos. Configuração do painel: a Fig. 1a mostra a configuração do painel balístico de $\mathrm{Al}_{2} \mathrm{O}_{3} / \mathrm{Al}$ com as peças descritas anteriormente: placa de apoio de alumínio 6063 (P1); placas de confinamento de alumina 92,2\% de pureza (P2); e uma placa alvo composta pela alumina $99,8 \%$ de pureza (P3). Entre as placas de alumina utilizou-se um espaçamento de $1,1 \mathrm{~mm}$, que foi preenchido com epóxi (Araldite Aralbras) para confinamento (P4). As placas cerâmicas foram fixadas ao suporte utilizado como material adesivo epóxi (Araldite Aralbras). As configurações das placas alvo são apresentadas na Fig. 1 b. $\mathrm{Na}$ Tabela I são apresentadas as configurações da placa de alumina de referência (experimento 1), das estruturas multicamadas à base de $\mathrm{Al}_{2} \mathrm{O}_{3}$ /epóxi e $\mathrm{Al}_{2} \mathrm{O}_{3}$ /epóxi/fibra de vidro (experimentos 4 e 7). A escolha da configuração do painel foi baseada nos resultados obtidos em [17]. Nesta referência as espessuras das placas de alumina foram de $8 \mathrm{~mm}$ e não apresentaram qualquer sinal de penetração na placa de apoio de alumínio [17]. Neste trabalho optou-se por confeccionar a placa de referência com espessura de 6 $\mathrm{mm}$ para provocar sinais de penetração que pudessem ser medidos, uma vez que a profundidade de penetração foi uma das formas adotadas para comparar os resultados. A escolha pelas dimensões da placa alvo de $50 \mathrm{~mm}$ foi a partir do cálculo do cone de fratura para a espessura de $6 \mathrm{~mm}$, conforme a equação [22]:

$$
\mathrm{L}_{\mathrm{C}}=\mathrm{d}_{\mathrm{p}}+2\left(\mathrm{e}_{\mathrm{c}} \cdot \operatorname{tg} 68^{\circ}\right)
$$

onde: $L_{C}$ - dimensão mínima da placa cerâmica $[\mathrm{m}]$; $\mathrm{d}_{\mathrm{P}}$ diâmetro do projétil [m]; $\mathrm{e}_{\mathrm{c}}$ - espessura da placa cerâmica [m]. Conforme a literatura [6], para uma configuração em mosaico a dimensão da placa cerâmica deve atender ao critério da capacidade de impacto, ou seja, deve possuir dimensões superiores ao do cone de fratura, de modo que o dano se limite à placa atingida.

Preparação dos tarugos de alumínio e placas de alumina: antes da montagem dos painéis, as superfícies de alumínio foram primeiramente lixadas, desengorduradas com tolueno durante 15 min e lavadas com detergente e água.

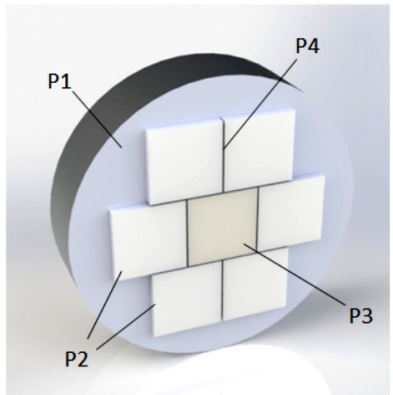

a)

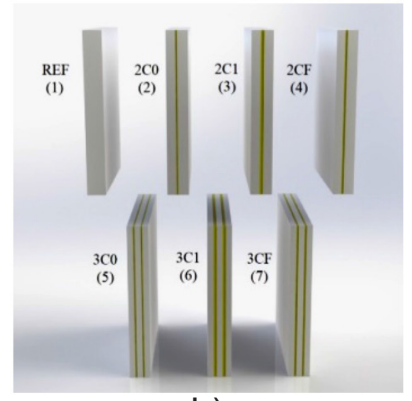

b)
Figura 1: Esquemas de: a) painel balístico $\mathrm{Al}_{2} \mathrm{O}_{3} / \mathrm{Al}$ (P1-placa de apoio; P2-placas de confinamento; P3-placa alvo; P4-rejunte de confinamento); e b) vista lateral da configuração das placas alvo. [Figure 1: Schematics of: a) ballistic panel $\mathrm{Al}_{2} \mathrm{O}_{3} / \mathrm{Al}$ ( $\mathrm{P} 1$-supporting plate; P2-confinement plates; P3-target plate; P4-containment grout; and b) side view of the target plates configuration.]
Tabela I - Configuração das placas cerâmicas alvo estudadas. [Table I - Configuration of the target ceramic plates studied.]

\begin{tabular}{ccccccc}
\hline Experimento & Amostra & $\mathrm{N}$ & $\begin{array}{c}\mathrm{L}_{1} \\
(\mathrm{~mm})\end{array}$ & $\begin{array}{c}\mathrm{L}_{2} \\
(\mathrm{~mm})\end{array}$ & $\begin{array}{c}\mathrm{L}_{3} \\
(\mathrm{~mm})\end{array}$ & $\begin{array}{c}\mathrm{W} \\
(\mathrm{mm})\end{array}$ \\
\hline 1 & $\mathrm{REF}$ & 1 & 6 & 0,0 & - & 6,0 \\
2 & $2 \mathrm{C} 0$ & 2 & 3 & 0,5 & - & 6,5 \\
3 & $2 \mathrm{C} 1$ & 2 & 3 & 1,0 & - & 7,0 \\
4 & $2 \mathrm{CF}$ & 2 & 3 & 0,5 & 0,15 & 6,65 \\
5 & $3 \mathrm{C} 0$ & 3 & 2 & 0,5 & - & 7,0 \\
6 & $3 \mathrm{C} 1$ & 3 & 2 & 1,0 & - & 8,0 \\
7 & $3 \mathrm{CF}$ & 3 & 2 & 0,5 & 0,15 & 7,3 \\
\hline
\end{tabular}

$N$ - número de placas de alumina empilhadas; $L_{1}$ - espessura das placas de alumina; $L_{2}$ - espessura das camadas de epóxi; $L_{3}$ - espessura do tecido de fibra de vidro; $w$ - espessura total das estruturas e espessura das placas de alumina de confinamento.

Depois de limpas e secas, a fim de formar pites e aumentar a rugosidade, as superfícies foram atacadas com solução de ácido sulfúrico (25\% de concentração volumétrica) e $67 \mathrm{~g} / \mathrm{L}$ de dicromato de sódio $\left(\mathrm{Na}_{2} \mathrm{Cr}_{2} \mathrm{O}_{7}\right)$ durante $15 \mathrm{~min}$ [21]. Após o tratamento químico os tarugos foram novamente lavados com detergente e água. As placas de alumina também foram desengorduradas com tolueno e lavadas, antes da montagem das estruturas multicamadas e dos mosaicos. A rugosidade superficial do alumínio foi determinada conforme a norma JIS B0601-1994, utilizando um rugosímetro Mitutoyo Surftest S-210. Foram realizadas medidas longitudinais e transversais ao sentido de corte em três corpos de prova, após o lixamento das superfícies.

Montagem das placas alvo multicamadas: as placas de alumina de 2 e $3 \mathrm{~mm}$ foram coladas com epóxi. A quantidade de adesivo para formar as camadas com as espessuras de 0,5 e 1,0 $\mathrm{mm}$ foi pesada diretamente sobre a placa de alumina. Para melhor controle da espessura, aplicou-se fita adesiva ao longo do perímetro das placas de alumina para formar uma barreira que impediu o escoamento do epóxi para além dos limites da placa. A massa foi espalhada com auxílio de uma espátula, formando uma camada uniforme. Na sequência, a segunda placa foi aplicada. Para se garantir boa fixação as placas foram levemente cisalhadas e pressionadas. No caso das estruturas com três camadas de alumina, a segunda camada de adesivo foi aplicada após a cura completa da primeira camada $(72 \mathrm{~h}$ ). Para as peças com aplicação de fibra de vidro, espalhou-se metade do epóxi sobre a peça, aplicouse o tecido, sendo então colocado o restante do epóxi. A fibra utilizada foi a do tipo E (Redelease).

Montagem do painel balístico: a preparação dos painéis compreendeu as seguintes etapas: montagem do mosaico cerâmico sobre um filme adesivo de $200 \times 200 \mathrm{~mm}^{2}$; espalhamento da camada adesiva sobre a placa de apoio; colagem do mosaico; remoção do filme adesivo; cura por $72 \mathrm{~h}$ na temperatura ambiente; preenchimento com epóxi dos espaçamentos entre as placas; cura por $72 \mathrm{~h}$. Durante a montagem do mosaico sobre o filme adesivo, foi utilizada fita adesiva dupla face transparente VHB 3M de aproximadamente $1,1 \mathrm{~mm}$ de espessura com o intuito de garantir homogeneidade 
no espaçamento entre as placas [17]. Espaçamentos maiores levam à maior vulnerabilidade da proteção e espaçamentos menores dificultam o preenchimento com o material de rejunte. A resina foi espalhada com o auxílio de uma espátula. Então o mosaico foi apoiado sobre a camada adesiva e pressionado a fim de distribuir bem o epóxi para evitar a formação de bolhas e garantir a homogeneidade da camada adesiva. Após a cura completa da camada adesiva (aproximadamente $72 \mathrm{~h}$ ), a fita adesiva foi completamente removida com o auxílio de solvente (tolueno) e os espaçamentos foram preenchidos com epóxi.

Caracterização das placas de alumina: foram realizados ensaios de caracterização para as aluminas de $99 \%$ de pureza (placas alvo). A densidade aparente das placas foi determinada pelo princípio de Arquimedes em água utilizando 3 amostras para cada espessura $(6,3$ e $2 \mathrm{~mm})$. A densidade relativa e a porosidade foram então calculadas por meio da relação entre densidade aparente e densidade real. Como densidade real da alumina- $\alpha$ considerou-se o valor de $3,98 \mathrm{~g} . \mathrm{cm}^{-3}$ [19]. O módulo de elasticidade foi determinado por meio da medida da velocidade de propagação do som no material (ATCP Sonelastic). O módulo de elasticidade está diretamente relacionado com a porosidade do material cerâmico, uma das correlações é descrita na equação [23]:

$$
\mathrm{E}_{\mathrm{c}}=\mathrm{E}_{2}\left(1-\mathrm{v}_{\mathrm{p}}^{23}\right)
$$

onde: $\mathrm{E}_{\mathrm{c}}$ - módulo de elasticidade $[\mathrm{Pa}] ; \mathrm{E}_{2}$ - módulo de elasticidade da fase densa $[\mathrm{Pa}] ; \mathrm{v}_{\mathrm{p}}$ - porosidade. A velocidade de propagação do som foi calculada utilizando a Eq. C [24], considerando um coeficiente de Poisson de 0,23 [17]. Para porosidades de até $0,12(12 \%)$ ocorre pouca variação no coeficiente de Poisson [25].

$$
\mathrm{V}=\sqrt{\frac{\mathrm{E}(1-\mathrm{v})}{\rho(1+v)(1-2 v)}}
$$

onde: V - velocidade de propagação do som $\left[\mathrm{m} . \mathrm{s}^{-2}\right] ; \mathrm{E}$ módulo de elasticidade [Pa]; $v$ - coeficiente de Poisson; @ - densidade $\left[\mathrm{kg} \cdot \mathrm{m}^{-3}\right]$. As resistências à flexão em três pontos das placas de alumina foram medidas utilizando-se uma máquina universal de ensaios mecânicos (Emic, DL10000). $\mathrm{O}$ ensaio de tenacidade à fratura $\left(\mathrm{K}_{\mathrm{IC}}\right)$ foi realizado por meio do ensaio de flexão em 3 pontos de peças entalhadas pelo método SENB (single-edge notched beam), com entalhe correspondendo a $40 \%$ da espessura do corpo de prova. A determinação da microdureza Knopp ocorreu utilizandose microdurômetro (Future Tech, FM 700), de acordo com a norma ASTM C1327-15. Para cada amostra foram consideradas 5 indentações válidas, com carga de $2 \mathrm{kgf}$ por $10 \mathrm{~s}$. A fragilidade e a capacidade de dissipação de energia balística estão relacionadas com as propriedades mecânicas do material cerâmico. Foram calculadas utilizando-se as equações [2]:

$$
\mathrm{B}=\frac{(\mathrm{H} \cdot \mathrm{E})}{\left(\mathrm{K}_{\mathrm{Ic}}^{2}\right)}
$$

$$
\mathrm{D}=0,36 \cdot \mathrm{B} \cdot \mathrm{c}
$$

onde: B - fragilidade $\left[\mathrm{m}^{-1}\right] ; \mathrm{D}$ - capacidade de dissipação de energia balística $\left[\mathrm{s}^{-1}\right] ; \mathrm{H}$ - microdureza Knoop [GPa]; E módulo de elasticidade [GPa]; c - velocidade de dissipação do som $[\mathrm{m} / \mathrm{s}] ; \mathrm{K}_{\mathrm{Ic}}$ - tenacidade à fratura $\left[\mathrm{MPa} \cdot \mathrm{m}^{1 / 2}\right]$.

Ensaio balístico: o desempenho balístico dos painéis foi avaliado por meio de ensaio de profundidade de penetração DOP (depth of penetration), de acordo com a norma NIJ0108.01 (National Institute of Justice). Os testes foram realizados no Centro de Avaliações do Exército (CAEx), do Centro Tecnológico do Exército (CTEx), pertencente ao exército brasileiro, na linha de ensaios para armas leves [26]. Durante os ensaios foram utilizados projéteis AP (perfurante) de 7,62x51 mm. Esta munição possui jaqueta de latão 70/30 e enchimento de chumbo na base. O núcleo é de aço SAE 4340 e apresenta dureza de 60 HRC, com dimensões de aproximadamente $24 \mathrm{~mm}$ de comprimento e $6,26 \mathrm{~mm}$ de diâmetro. A massa do projétil foi de $9,8 \mathrm{~g}$. As munições foram disparadas a partir de um provete. A velocidade do projétil foi medida com o auxílio de um sensor óptico, a uma distância de $3 \mathrm{~mm}$ do alvo. A energia cinética média utilizada no ensaio balístico foi calculada com base na velocidade média de ensaio e massa do projétil. Os ensaios foram realizados em triplicata - para cada configuração estudada foram montados três painéis. Como referência de eficiência balística para as placas de apoio sem proteção, foi utilizada uma profundidade de penetração de $63 \pm 4 \mathrm{~mm}$ [17]. Para calibrar a mira foram realizados três disparos sequenciais contra o tarugo de alumínio. Observou-se que os disparos não atingiram o mesmo ponto no alvo, devido aos desvios de trajetória dos projéteis. A distância média entre centros das perfurações nos três disparos foi de 15,6 $\pm 0,7 \mathrm{~mm}$. Após o ensaio balístico, o desempenho dos painéis foi avaliado a partir de três parâmetros: 1) $\mathrm{d}_{\text {cone }}$ - diâmetro do cone de fratura; 2) DOP - profundidade de penetração do projétil; e 3) d - distância do ponto atingido pelo projétil em relação ao centro da placa alvo.

\section{RESULTADOS E DISCUSSÃO}

Propriedades da alumina: na Tabela II são apresentadas as propriedades das placas de alumina. Os valores de densidade aparente encontrados foram semelhantes. $\mathrm{O}$ processamento de placas de diferentes espessuras não causou variação significativa nas propriedades mecânicas, tais como módulo de elasticidade, tenacidade à fratura e microdureza. A resistência mecânica à flexão reduziu com a redução da espessura da alumina. Pelo fato de não terem sido observadas variações significativas do módulo de elasticidade e da tenacidade à fratura, a variação foi atribuída ao aumento do tamanho do defeito natural com a redução da espessura. Valores de tamanho de defeito natural de aproximadamente $90 \mu \mathrm{m}$ foram medidos em [27] para a mesma alumina empregada neste trabalho, porém com espessura de 5 $\mathrm{mm}$. Atribuiu-se este resultado principalmente à etapa de conformação por prensagem. Jiusti et al. [17] utilizaram uma 
alumina com módulo de elasticidade de $337 \mathrm{GPa}$, tenacidade à fratura de 3,6 MPa.mº,5 e microdureza Knoop de 14,2 GPa. As propriedades das aluminas utilizadas neste trabalho foram semelhantes à alumina utilizada em [17]. O módulo de elasticidade e a dureza são as propriedades mecânicas de maior significância para a resistência balística [28]. Pôdese observar que as propriedades foram equivalentes para as condições de alumina testadas. A fragilidade e a capacidade de dissipação de energia balística são relações entre as propriedades mecânicas dos materiais. Tais propriedades têm influência na propagação das ondas de choque durante o impacto balístico, afetando diretamente o desempenho do material [2]. Os valores de fragilidade (B) e capacidade de dissipação de energia balística (D) foram semelhantes para as três configurações, uma vez que tais propriedades não dependem da espessura da amostra. Os resultados encontrados em [17], tratando-se das placas de mosaico, foi $B=4.10^{8} \mathrm{~m}^{-1}$ e $\mathrm{D}=1,5.10^{12} \mathrm{~s}^{-1}$. Tomando-se como base a alumina de $6 \mathrm{~mm}$, esperava-se uma performance balística levemente superior neste trabalho.

Suporte de alumínio: antes do tratamento térmico, os

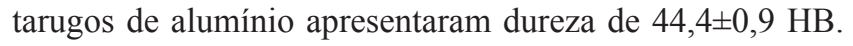
Após o tratamento, a dureza do alumínio passou para $74 \pm 8$ HB. A rugosidade superficial medida na direção transversal

Tabela II - Propriedades mecânicas das placas de aluminas de 6, 3 e $2 \mathrm{~mm}$ de espessura.

[Table II - Mechanical properties of alumina plates with 6, 3 and $2 \mathrm{~mm}$ in thickness.]

\begin{tabular}{cccc}
\hline Propriedade & $\begin{array}{c}\text { Alumina } \\
6 \mathrm{~mm}\end{array}$ & $\begin{array}{c}\text { Alumina } \\
3 \mathrm{~mm}\end{array}$ & $\begin{array}{c}\text { Alumina } \\
2 \mathrm{~mm}\end{array}$ \\
\hline $\mathbf{Q}_{\text {aparente }}\left(\mathrm{g} \cdot \mathrm{cm}^{-3}\right)$ & 3,85 & 3,84 & 3,82 \\
$\mathbf{Q}_{\text {relativa }}(\%)$ & 96,7 & 96,5 & 96,0 \\
Porosidade $(\%)$ & 3,3 & 3,5 & 4,0 \\
$\mathrm{E}(\mathrm{GPa})$ & 329 & 321 & 320 \\
$\mathrm{~V}_{\text {som }}\left(\mathrm{m} \cdot \mathrm{s}^{-1}\right)$ & 9945 & 9850 & 9865 \\
$\mathrm{~K}_{\mathrm{Ic}}\left(\mathrm{MPa} \cdot \mathrm{m}^{1 / 2}\right)$ & 3,8 & 3,7 & 3,7 \\
$\mathrm{Microdureza}(\mathrm{GPa})$ & 11,6 & 13,6 & 14,1 \\
$\sigma_{\text {flexão }}(\mathrm{MPa})$ & 247 & 189 & 166 \\
$\mathrm{a}^{*}(\mu \mathrm{m})$ & 60 & 98 & 127 \\
$\mathrm{~B}\left(10^{-8} \mathrm{~m}^{-1}\right)$ & 2,64 & 3,09 & 3,20 \\
$\mathrm{D}\left(10^{-12} \mathrm{~s}^{-1}\right)$ & 0,95 & 1,10 & 1,14 \\
\hline
\end{tabular}

*-tamanho do defeito natural.

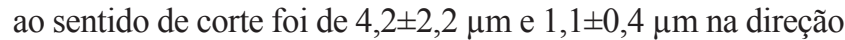
longitudinal. Estes valores foram próximos aos obtidos em [17], na qual foram utilizados tarugos com dureza de aproximadamente $73 \mathrm{HB}$ e rugosidade de 4,6 $\mu \mathrm{m}$ na direção transversal e $0,4 \mu \mathrm{m}$ na direção longitudinal.

Desempenho balístico: na Tabela III são apresentadas a velocidade média e a energia cinética inicial dos projéteis durante o ensaio balístico para cada condição experimental. De acordo com a norma NIJ 0108.01 para o nível IV a velocidade do projétil deve ser de $868 \pm 15 \mathrm{~m} / \mathrm{s}$. Os valores de velocidade foram similares ao especificado pela norma [29]. Os resultados do ensaio balístico dos 21 painéis preparados são apresentados na Tabela IV. Conforme mostrado na Fig. 2 , as placas de referência apresentaram melhor resistência balística, pois a profundidade de penetração foi menor comparada aos sistemas multicamadas. No caso das aluminas de referência, as amostras REF/A e REF/B apresentaram DOP similares. No entanto, o distanciamento do ponto atingindo pelo projétil em relação ao centro da placa alvo reduziu o desempenho balístico. A placa que foi atingida mais ao centro $(\mathrm{d}=3,96 \mathrm{~mm})$ apresentou a menor DOP $(\sim 0)$. $\mathrm{O}$ resultado para a placa de referência corroborou o melhor desempenho esperado para as peças produzidas neste trabalho em relação aos ensaiados em [17], com peças de 8,0 $\mathrm{mm}$ de espessura. No caso das estruturas multicamadas, o aumento do número de camadas resultou na diminuição da resistência balística. As estruturas de 2 camadas de alumina e epóxi apresentaram DOP entre 12 e $17 \mathrm{~mm}$, enquanto que a DOP das estruturas de 3 camadas e epóxi foi de 17 a 33 mm (Tabela IV). Os resultados balísticos demonstraram que para todas as condições experimentais a dispersão entre os resultados teve alguma correlação, embora fraca, com a posição que o projétil atingiu o alvo. Pode-se observar na Fig. 3 que o distanciamento do ponto atingido pelo projétil em relação ao centro da placa alvo reduziu o desempenho balístico.

Nas Figs. 2 e 4 são mostrados os painéis multicamadas de $\mathrm{Al}_{2} \mathrm{O}_{3}$ /epóxi após o ensaio balístico e comparação com o painel de referência. No caso do painel de referência observou-se que o dano se restringiu à placa alvo e não houve penetração no alumínio. A área do cone de fratura teve praticamente as mesmas dimensões da placa. O sistema multicamada, mesmo mantendo inalterada a espessura total da alumina, piorou significativamente o grau de proteção balística do conjunto. Por outro lado, com o aumento do número de camadas ocorreu uma diminuição na propagação dos danos radiais da placa cerâmica, pois o diâmetro do cone

Tabela III - Velocidade média $\left(\mathrm{V}_{\mathrm{m}}\right)$ e energia cinética inicial (E) do projétil durante ensaio balístico, para cada condição experimental.

[Table III - Average velocity $\left(V_{m}\right)$ and initial kinetic energy (E) of the projectile during ballistic test, for each experimental condition.]

\begin{tabular}{cccccccc}
\hline Amostra & REF & $2 \mathrm{C} 0$ & $2 \mathrm{C} 1$ & $2 \mathrm{CF}$ & $3 \mathrm{C} 0$ & $3 \mathrm{C} 1$ & $3 \mathrm{CF}$ \\
\hline $\mathrm{V}_{\mathrm{m}}(\mathrm{m} / \mathrm{s})$ & $866 \pm 6$ & $866 \pm 9$ & $864 \pm 8$ & $869 \pm 14$ & $868 \pm 14$ & $877 \pm 12$ & $866 \pm 9$ \\
$\mathrm{E}(\mathrm{J})$ & $3638 \pm 46$ & $3638 \pm 77$ & $3623 \pm 71$ & $3658 \pm 118$ & $3651 \pm 118$ & $3726 \pm 101$ & $3639 \pm 74$ \\
\hline
\end{tabular}


Tabela IV - Resultados do ensaio balístico das aluminas monolíticas e das estruturas multicamadas estudadas.

[Table IV - Results of the ballistic test of monolithic aluminas and multilayer structures studied.]

\begin{tabular}{|c|c|c|c|}
\hline Amostra & $\mathrm{d}_{\text {cone }}(\mathrm{mm})$ & $\mathrm{d}(\mathrm{mm})$ & $\mathrm{DOP}(\mathrm{mm})$ \\
\hline REF/A & 55,9 & 13,0 & 2,3 \\
\hline $\mathrm{REF} / \mathrm{B}$ & 52,5 & 11,3 & 2,4 \\
\hline $\mathrm{REF} / \mathrm{C}$ & 59,8 & 4,0 & 0,0 \\
\hline $2 \mathrm{C} 0 / \mathrm{A}$ & 57,4 & 12,9 & 11,9 \\
\hline $2 \mathrm{C} 0 / \mathrm{B}$ & 63,6 & 7,8 & 8,9 \\
\hline $2 \mathrm{C} 0 / \mathrm{C}$ & 70,7 & 14,1 & 13,5 \\
\hline $2 \mathrm{C} 1 / \mathrm{A}$ & 51,9 & 10,0 & 13,8 \\
\hline $2 \mathrm{C} 1 / \mathrm{B}$ & 59,4 & 5,3 & 12,6 \\
\hline $2 \mathrm{C} 1 / \mathrm{C}$ & $-*$ & 20,4 & 12,0 \\
\hline $2 \mathrm{CF} / \mathrm{A}$ & 70,7 & 7,3 & 11,8 \\
\hline $2 \mathrm{CF} / \mathrm{B}$ & 49,1 & 11,2 & 16,7 \\
\hline $2 \mathrm{CF} / \mathrm{C}$ & - & 23,1 & 15,6 \\
\hline $3 \mathrm{C} 0 / \mathrm{A}$ & 48,5 & 6,8 & 25,4 \\
\hline $3 \mathrm{C} 0 / \mathrm{B}$ & 45,9 & 13,3 & 17,0 \\
\hline $3 \mathrm{C} 0 / \mathrm{C}$ & $-*$ & 16,8 & 29,8 \\
\hline $3 \mathrm{C} 1 / \mathrm{A}$ & $-*$ & 27,1 & 33,2 \\
\hline $3 \mathrm{C} 1 / \mathrm{B}$ & 61,4 & 5,4 & 23,2 \\
\hline $3 \mathrm{C} 1 / \mathrm{C}$ & 49,3 & 10,8 & 20,3 \\
\hline $3 \mathrm{CF} / \mathrm{A}$ & 51,8 & 16,6 & 32,9 \\
\hline $3 \mathrm{CF} / \mathrm{B}$ & 58,7 & 8,2 & 21,6 \\
\hline $3 \mathrm{CF} / \mathrm{C}$ & - & 24,6 & 21,7 \\
\hline
\end{tabular}

$\overline{d_{\text {cone }} \text { - diâmetro do cone de fratura; } d \text { - distância do ponto atingido pelo }}$ projétil em relação ao centro da placa alvo; DOP - profundidade de penetração do projétil; * - não foi possível medir devido ao desprendimento das placas do mosaico.

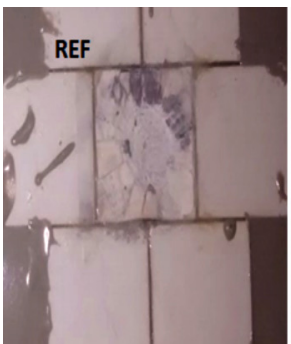

a)

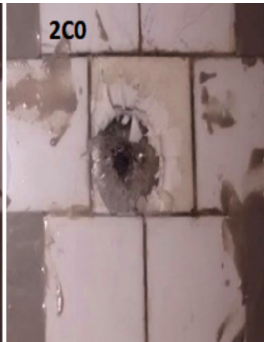

b)

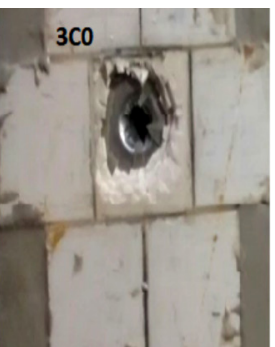

c)
Figura 2: Imagens dos painéis após ensaio balístico.

[Figure 2: Images of the panels after ballistic test.]

de fratura foi reduzido. De acordo com a Eq. A, o cone de fratura reduz com a redução da espessura da amostra. Os resultados obtidos sugerem que o sistema multicamada não se comportou como um sistema único, mas sim individualizado, ou seja, o mecanismo de dissipação de fratura esperado não surtiu efeito para o sistema multicamada elaborado. Por outro lado, a baixa dissipação da energia do impacto na primeira placa atingida indicou que uma fratura predominantemente

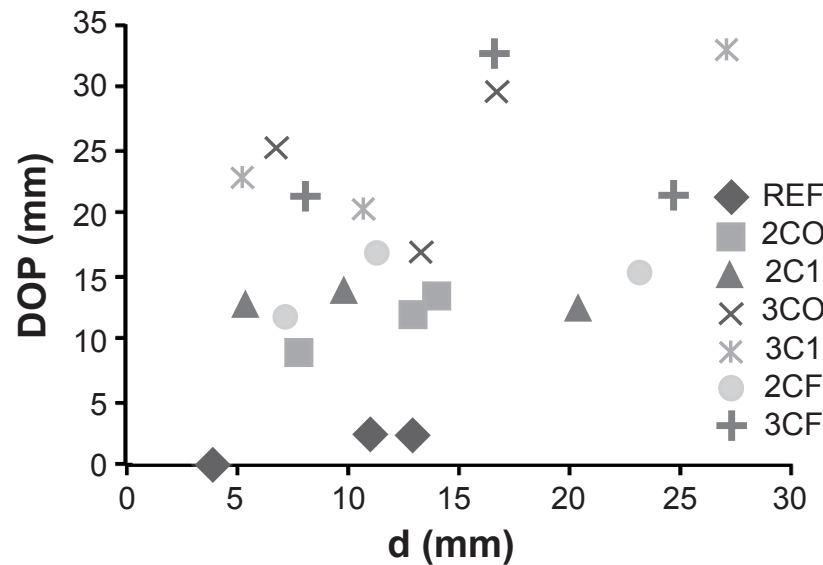

Figura 3: Profundidade de penetração (DOP) vs. distância a partir do centro (d).

[Figure 3: Depth of penetration (DOP) vs. distance from the center (d).]

por flexão tenha ocorrido. Isso levou a uma redução na capacidade erosiva da cerâmica sobre o projétil e maior energia residual para as camadas seguintes e para a placa de apoio. Os dados de profundidade de penetração (DOP) plotados em função da espessura da camada de alumina são apresentados na Fig. 5a. A variação da DOP com a variação da espessura da camada de epóxi é apresentada na Fig. 5b. Observou-se redução assintótica, tendendo a zero, da DOP com o aumento da espessura da alumina. O efeito da variação da espessura do epóxi foi menos pronunciado; apesar de existir uma grande variabilidade nos dados, observou-se apenas um sutil aumento na DOP com as variações de 0,5 para 1,0 $\mathrm{mm}$ de espessura. É muito provável que mesmo uma espessura de $0,5 \mathrm{~mm}$ seja grande o suficiente para o sistema comportar-se de modo semelhante a uma camada de 1,0 $\mathrm{mm}$. Nestes dois casos, aumentou a quantidade de fraturas por esforço de flexão da placa atingida.

Por meio de análise de variância e regressão linear foi verificada a significância estatística dos fatores estudados sobre o desempenho balístico dos painéis. Os fatores, transformados em regressores, foram rotulados como:

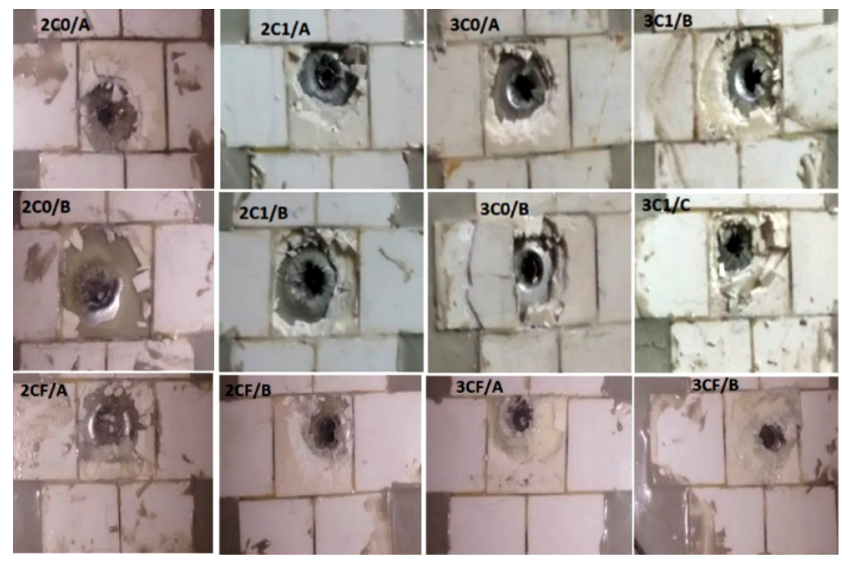

Figura 4: Imagens de painéis multicamadas de $\mathrm{Al}_{2} \mathrm{O}_{3}$ /epóxi após ensaio balístico.

[Figure 4: Images of multilayer panels of $\mathrm{Al}_{2} \mathrm{O}_{3}$ /epoxy after ballistic test.] 

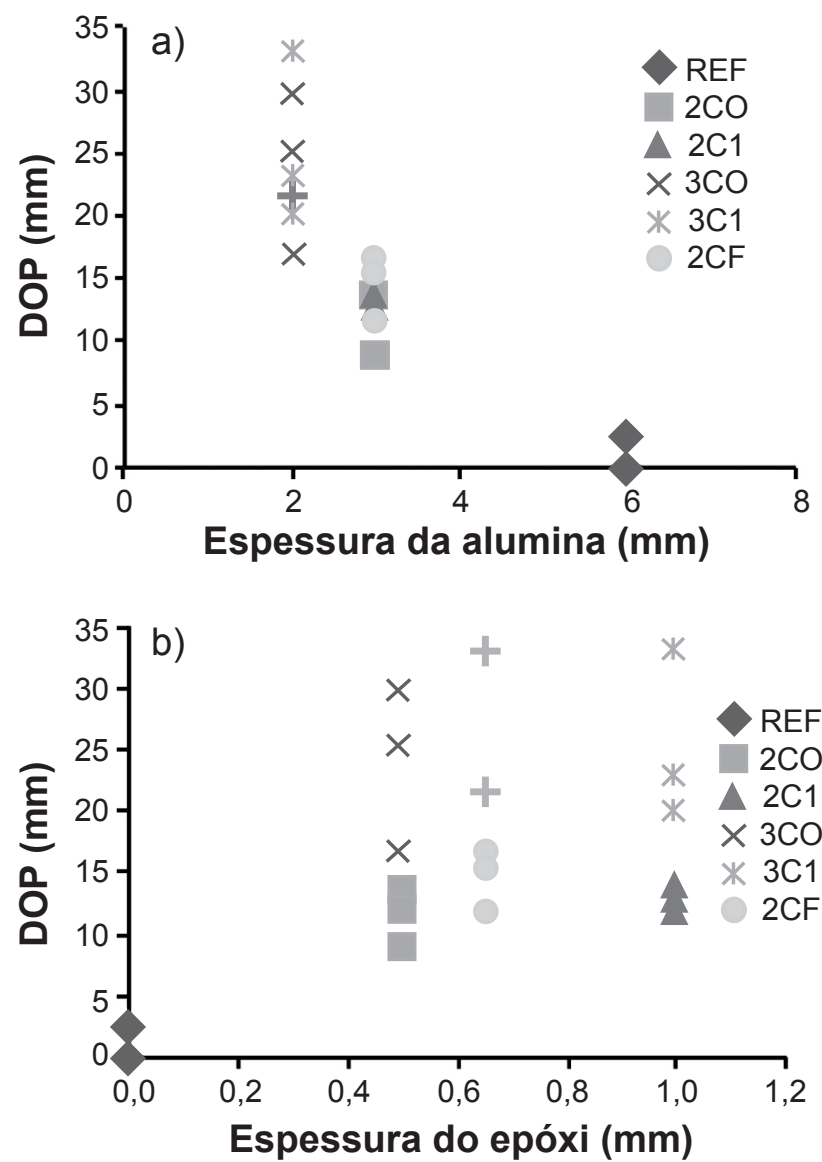

Figura 5: Profundidade de penetração (DOP) em função da espessura de: a) alumina; e b) epóxi.

[Figure 5: Depth of penetration (DOP) as a function of thickness of: a) alumina; and b) epoxy.]

velocidade do projétil (X1); espessura da alumina (X2); efeito quadrático da espessura da alumina (X2.X2); distância do ponto de impacto em relação ao centro da placa (X3); espessura da camada de epóxi (X4); e adição de fibra de vidro (X5). Os resultados da análise estatística são apresentados na Tabela V. O valor F de significação geral do modelo resultou em valor de 4,4.10-6 correspondendo a um valor aceitável. Considerando-se um nível de significância estatística de $95 \%$ (valor $\mathrm{P}<0,05$ ) pôde-se verificar que os fatores espessura da camada de epóxi e adição de fibra tiveram efeitos pouco significativos, portanto, podem ser desconsiderados como regressores do modelo. A Tabela VI mostra o resultado estatístico da regressão e teste de significância para o novo modelo. Neste caso todos os regressores apresentaram efeito significativo. $\mathrm{O}$ valor $\mathrm{F}$ de significação resultou em $1,58 \cdot 10^{-7}$, sugerindo que a simplificação no modelo não ocasionou prejuízos em sua previsibilidade. A espessura da placa de alumina apresentou efeito linear (X2) e quadrático (X2.X2) como sendo significativos. O coeficiente positivo para $\mathrm{X} 1$ indicou que quanto maior a velocidade do projétil maior é a profundidade de penetração, o que é fisicamente coerente e justifica a medida de velocidade a cada disparo. Da mesma forma a distância do ponto de impacto em relação ao centro da placa
(X3), ou seja, mais perto da borda maior é a vulnerabilidade da proteção e maior a DOP. O parâmetro mais relevante foi a espessura da placa de alumina. O coeficiente fortemente negativo indicou não apenas que quanto maior a espessura da alumina menor é a DOP como também que este efeito é determinante da eficiência do sistema.

Tabela V - Resultados da regressão linear para avaliar o efeito das variáveis analisadas no desempenho balístico.

[Table $V$ - Results of linear regression to evaluate the effect of the analyzed variables on ballistic performance.]

\begin{tabular}{ccccc}
\hline & Coeficiente & $\begin{array}{c}\text { Erro } \\
\text { padrão }\end{array}$ & Stat t & Valor-P \\
\hline Interseção & $-111,83$ & 77,04 & $-1,45$ & 0,169 \\
X1 & 0,19 & 0,09 & 2,18 & 0,047 \\
X2 & $-20,26$ & 4,64 & $-4,37$ & 0,001 \\
X2.X2 & 1,90 & 0,61 & 3,11 & 0,008 \\
X3 & 0,27 & 0,13 & 2,06 & 0,059 \\
X4 & 0,89 & 4,25 & 0,21 & 0,837 \\
X5 & 8,84 & 14,25 & 0,62 & 0,545 \\
\hline
\end{tabular}

Tabela VI - Resultados da regressão linear desconsiderando o efeito da espessura da camada de epóxi e adição de fibra de vidro.

[Table VI - Results of linear regression disregarding the effect of epoxy layer thickness and fiber glass addition.]

\begin{tabular}{ccccc}
\hline & Coeficiente & $\begin{array}{c}\text { Erro } \\
\text { padrão }\end{array}$ & Stat t & Valor-P \\
\hline Interseção & $-111,89$ & 72,40 & $-1,55$ & 0,140 \\
X1 & 0,19 & 0,08 & 2,33 & 0,033 \\
X2 & $-19,77$ & 4,17 & $-4,75$ & 0,0002 \\
X2.X2 & 1,81 & 0,51 & 3,53 & 0,003 \\
X3 & 0,29 & 0,12 & 2,35 & 0,032 \\
\hline
\end{tabular}

Na Fig. 6 é apresentado o gráfico dos valores observados em função dos valores previstos pelo modelo de regressão. Verifica-se dispersão aleatória dos dados experimentais em torno da bissetriz do gráfico. Por outro lado, observa-se uma tendência de aumentar a dispersão dos resultados à medida que aumenta a profundidade de penetração. Observa-se na Fig. 6 que existem três agrupamentos de dados: um com DOP inferior a $5 \mathrm{~mm}$, o segundo entre 10 e $15 \mathrm{~mm}$ e o terceiro entre 20 e $35 \mathrm{~mm}$. Para cada agrupamento o desvio médio foi calculado e correlacionado com a profundidade de penetração média. $\mathrm{O}$ resultado está apresentado na Fig. 7. Nesta figura também está inserida a informação do desvio médio da DOP medida em [17] para o tarugo de alumínio sem as placas de alumina de proteção. Observa-se que o desvio médio aumenta com o aumento da profundidade de penetração. Os resultados sugerem uma tendência consistente dos valores tenderem ao desvio médio da placa de alumínio. Este resultado é factível, uma vez que o modelo 


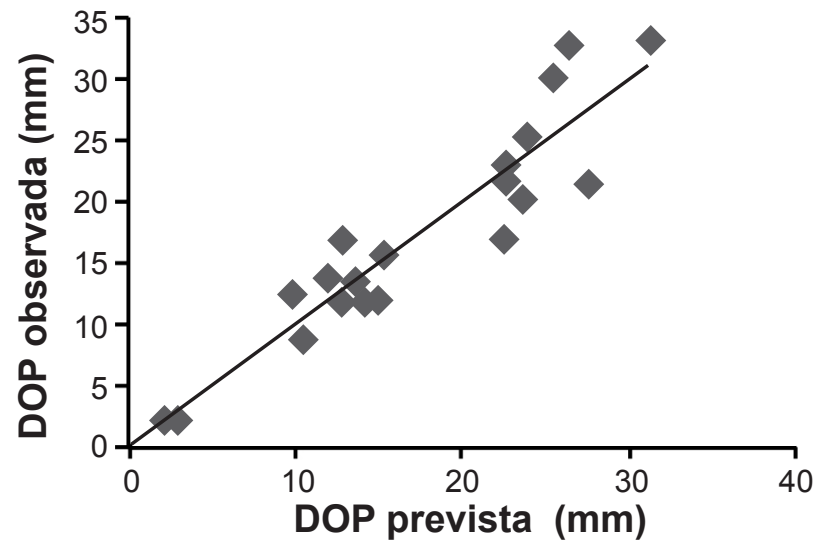

Figura 6: Resultados de DOP prevista vs. DOP observada. [Figure 6: Results of the predicted DOP vs. observed DOP.]

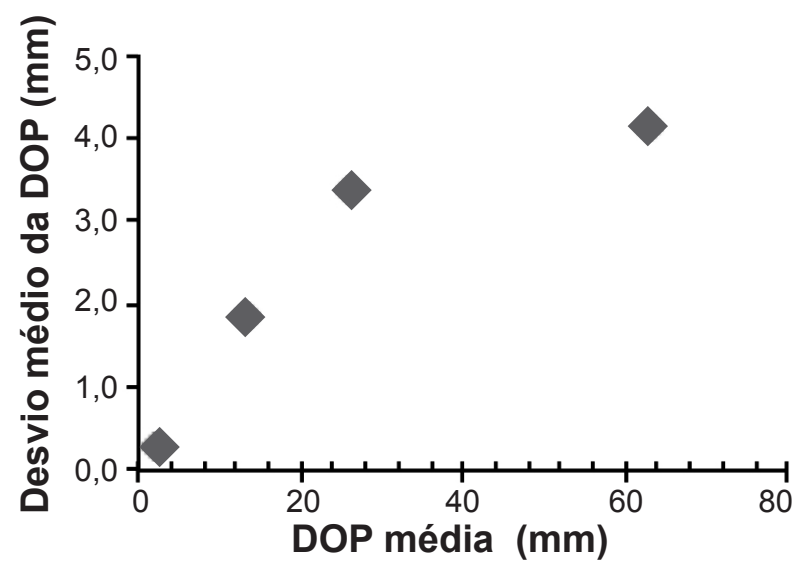

Figura 7: Desvio médio da DOP vs. DOP média. [Figure 7: Mean deviation of DOP vs. average DOP.]

de regressão adotado não levou em consideração nenhum parâmetro relativo à placa de apoio. À medida que aumenta a DOP maior é o efeito do alumínio na absorção da energia cinética do projétil.

\section{CONCLUSÕES}

Estruturas multicamadas alumina/epóxi para aplicação balística foram obtidas e testadas em comparação à alumina de camada única em painéis alumina/alumínio. $O$ ensaio de profundidade de penetração (DOP) foi utilizado para a determinação do desempenho balístico. As propriedades mais relevantes no desempenho balístico, ou seja, a dureza e o módulo de elasticidade foram equivalentes para as diferentes espessuras de alumina testada. Isto indicou que as variáveis de processamento empregadas para produzir peças com diferentes espessuras não alteraram as características intrínsecas do material. Por outro lado, o tamanho do defeito natural aumentou com a redução da espessura das peças compactadas a partir do pó em prensagem uniaxial. $\mathrm{O}$ fator mais relevante no desempenho balístico foi a espessura das placas de alumina. Mesmo que a espessura total do conjunto de placas de alumina tenha permanecido a mesma, as placas com espessura individual de $6,0 \mathrm{~mm}$ foram as únicas com desempenho satisfatório. A análise do cone de fratura sugeriu que as placas de alumina no sistema multicamada se comportaram de modo individual e não como um sistema único, sugerindo fratura com maior predominância de esforço de flexão. A espessura do epóxi entre 0,5 e 1,0 mm não demostrou ser uma variável significativa. Apesar disso espessuras $<0,5 \mathrm{~mm}$ poderiam levar à melhoria do comportamento global do sistema multicamada devido à tendência de reduzir os efeitos de flexão. $O$ maior distanciamento do ponto atingindo pelo projétil em relação ao centro da placa alvo reduziu o desempenho balístico em sistemas de mosaico. A análise estatística indicou ainda que as variações na velocidade do projétil apresentaram efeito significativo sobre o desempenho balístico, muito embora tenha sido mantido aproximadamente constante o carregamento de pólvora da munição. O fato de ter sido estratificado o efeito deste fator permitiu apurar a significância do efeito do ponto atingido pelo projétil na placa. A adição de fibra de vidro não aparentou efeito significativo sobre o desempenho balístico. Eventualmente uma trama mais aberta, do tipo E, por exemplo, traria melhores resultados.

\section{AGRADECIMENTOS}

Ao CAEx (Exército Brasileiro) por seu apoio com os ensaios balísticos e à Coordenação de Aperfeiçoamento de Pessoal de Nível Superior (CAPES/Brasil, Edital PróDefesa n.031/2013) por financiar este trabalho.

\section{REFERÊNCIAS}

[1] J. Lopez-Puente, A. Arias, R. Zaera, C. Navarro, Int. J. Impact Eng. 32 (2005) 321.

[2] E. Medvedovski, Ceram. Int. 36 (2010) 2103.

[3] W. Liu, Z. Chen, X. Cheng, Y. Wang, X. Chen, J. Liu, B. Li, S. Wang, Mater. Design 87 (2015) 421.

[4] I.G. Crouch, M. Kesharaju, R. Nagarajah, Ceram. Int. 41 (2015) 11581.

[5] M.V. Silva, D. Stainer, H.A. Al-Qureshi, D. Hotza, Cerâmica 60 (2014) 323.

[6] C.A.O. Couto, "Estudo de blindagem para proteção contra impactos de micrometeoróides em satélites artificiais", Dis. Mestr., INPE, S. José Campos (2011).

[7] R. Zaera, S. Sánchez-Sáez, J.L. Pérez-Castellanos, C. Navarro, Composites Part A 31 (2000) 823.

[8] S. Yadav, G. Ravichandran, Int. J. Impact Eng. 28 (2003) 557.

[9] K. Akella, Procedia Eng. 173 (2017) 244.

[10] A.V. Ignatova, S.B. Sapozhnikov, N. Yu. Dolganina, Int. J. Mech. Sci. 131-132 (2017) 672.

[11] I. Crouch, in "The science of armour materials", I. Crouch (Ed.), Armour Sol. Pty, Victoria (2017) 675.

[12] F.D. Minatto, "Compósito cerâmico multicamada alumina/LZSA: estudo das composições das camadas", Dis. Mestr., UNESC, Criciúma (2015).

[13] P.J. Hazell, C.J. Roberson, M. Moutinho, Mater. Design 29 (2008) 1497. 
[14] E. Medvedovski, Ceram. Int. 36 (2010) 2117.

[15] M. Grujicic, B. Pandurangan, B. d'Entremont, Mater. Design 41 (2012) 380.

[16] Z.H. Tan, X. Han, W. Zhang, S.H. Luo, Int. J. Impact Eng. 37 (2010) 1162.

[17] J. Jiusti, E.H. Kammer, L. Neckel, N.J. Lóh, W. Trindade, A.O. Silva, O.R.K. Montedo, A. De Noni Jr., Ceram. Int. 43 (2017) 2697.

[18] L.R. Vyshnyakov, A.V. Mazna, A.V. Neshpor, V.A. Kokhanyi, O.N. Oleksyuk, Strength Mater. 36 (2004) 643.

[19] D.C.N. Fabris, "Efeito da adição de precursor vitrocerâmico de cordierita na densificação e nas propriedades mecânicas de alumina para uso balístico", Dis. Mestr., UNESC, Criciúma (2017).

[20] M. Übeyli, R.O. Yildirim, B. Ögel, J. Mater. Proces. Technol. 196 (2008) 356.

[21] ASM Handb., v.8, "Mechanical testing and evaluation", ASM Int. (2000).
[22] R.L. Woodward, Int. J. Impact Eng. 9 (1990) 455.

[23] Y. Hirata, T. Shimonosono, Ceram. Int. 21 (2016) 3014.

[24] L.S. Chang, T.-H. Chuang, W.J. Wei, Mater. Charact. 45 (2003) 221.

[25] M. Asmani, C. Kermel, A. Leriche, M. Ourak, J. Eur. Ceram. Soc. 21 (2001) 1081.

[26] W. Trindade, "Influência da geometria e da microestrutura no comportamento dinâmico da alumina aditivada com nióbia", Tese Dr., Inst. Militar Eng., Rio Janeiro (2012).

[27] O.R.K. Montedo, P.C. Milak, C.A. Faller, M. Peterson, A. De Noni Jr., Mater. Res. 21 (2018) 1.

[28] A. Krell, E. Strassburger, Mater. Sci. Eng. A 597 (2014) 422.

[29] Nat. Inst. Justice, "Ballistic resistant protective materials", 0108.01 (EUA) 1985.

(Rec. 28/02/2018, Rev.06/07/2018, 25/09/2018, 13/11/2018, Ac. 25/11/2018) 